\title{
Prenatal Stress Selectively Impairs Neuroligin 1- Dependent Neurogenesis by Suppressing Astrocytic FGF2-Neuronal FGFR1 Axis
}

Gee Euhn Choi

Seoul National University College of Veterinary Medicine

Chang Woo Chae

Seoul National University College of Veterinary Medicine

Mo Ran Park

Seoul National University College of Veterinary Medicine

Jee Hyeon Yoon

Seoul National University College of Veterinary Medicine

Young Hyun Jung

Seoul National University College of Veterinary Medicine

Hyun Jik Lee

Chungbuk National University

Ho Jae Han ( $\nabla$ hjhan@snu.ac.kr )

Seoul National University College of Veterinary Medicine https://orcid.org/0000-0002-0657-1766

\section{Research Article}

Keywords: Prenatal stress, Glucocorticoid, Neurogenesis, Glutamatergic synaptogenesis, AstrocyteNeuron interaction, Neuroligin 1

Posted Date: January 7th, 2022

DOI: https://doi.org/10.21203/rs.3.rs-1201112/v1

License: (a) (1) This work is licensed under a Creative Commons Attribution 4.0 International License. Read Full License 


\section{Abstract}

Exposure to maternal stress irreversibly impairs neurogenesis of offspring through inducing life-long effects on interaction between neurons and glia under raging differentiation process, culminating in cognitive and neuropsychiatric abnormalities in adulthood. We identified how prenatal exposure to the stress-hormone glucocorticoid impairs synapse formation and subsequent neurogenesis using human induced pluripotent stem cell (iPSC)-derived neural stem cell (NSC) and ICR mice. Following prenatal glucocorticoid exposure, NSC-derived astrocytes were found to be A1-like neurotoxic astrocytes. Moreover, cortisol-treated astrocyte conditioned media (ACM) then specifically downregulated AMPA receptormediated glutamatergic synaptic formation and transmission in differentiating neurons, by inhibiting localization of ionotropic glutamate receptor (GluR) 1/2 into synapses. We revealed that downregulated astrocytic fibroblast growth factor 2 (FGF2) and nuclear fibroblast growth factor receptor 1 (FGFR1) of neurons are key pathogenic factors for reducing glutamatergic synapse formation, according to data from RNA sequencing and antibody array. We further confirmed that cortisol-treated ACM specifically decreased the binding of neuronal FGFR1 to the synaptogenic NLGN1 promoter, but this was reversed by FGFR1 restoration. Upregulation of neuroligin 1, which is important in scaffolding GluR1/2 into the postsynaptic compartment, eventually normalized glutamatergic synaptogenesis and subsequent neurogenesis. Moreover, FGF2 pretreatment of a prenatal corticosterone-exposed mouse elevated neuroligin 1 expression and trafficking of GluR1/2 into the postsynaptic compartment, improving spatial memory and depression/anxiety-like behaviors. In conclusion, we demonstrated that neuroligin 1 restoration by astrocytic FGF2 and its downstream neuronal nuclear FGFR1 as a critical target of prenatal stress-induced glutamatergic synaptogenesis and demonstrated its function in controlling both neurogenesis and hippocampal-related behaviors.

\section{Introduction}

Stress is a high risk factor for suppressing the development and differentiation of both embryonic and adult neural stem cell (NSC). While the effect of stress during adulthood on neural tissue is usually reversible, prenatal or early-postnatal stress continues to adversely affect neurogenesis throughout life by permanently downregulating neurogenic or neurotrophic factors [1-3]. As a result, rodents or primates that have experienced prenatal stress often develop neuropsychiatric or cognitive disorders as juveniles or adolescents even if the stress has not continued [4-6]. Glucocorticoid largely mediates these deleterious effects of prenatal stress and is most influential in the hippocampus, the center for emotion and memory formation, of both in animals and humans due to its abundance of glucocorticoid receptor (GR) [7]. However, the transcription of GR differs depending on the maturity of neurons in that GR is not transiently expressed at the certain stage of immature neurons. Therefore, other glucocorticoid-mediated crosstalk pathways that are independent from GR can largely affect neural differentiation during early life $[2,8]$. Furthermore, prenatal stress evokes significant changes to epigenetic profiles through DNA methylation/demethylation or chromatin remodeling, which last throughout life $[9,10]$. These specific changes due to prenatal stress suggest that they are unique to fetus or infant brains, in which 
neurogenesis and gliosis rigorously occur, but very little is known about the underlying molecular and cellular alterations.

In adults, neuroprogenitor cell (NPC), which occupies small portions of neuronal cells, mostly derived from the subgranular zone (SGZ) of the hippocampus undergo gliosis to buffer stress responses by triggering neurogenesis and strengthening brain circuits [11]. Astrocytes, the most abundant glial cell type, interact with neurons especially by neurotrophic support to maintain CNS health during stress by aiding neurogenesis, synapse formation, and neural tissue repair $[12,13]$. However, large portions of NPC can be extensively affected by damaging effect of prenatal stress on astrocytes. Neurotrophic support by astrocytes is a determining factor in outcome of mid- to late-stage prenatal neurogenesis, because gliosis peaks during the second week of rodent fetal life, when the levels of several neurotrophic factors such as FGF2 and BDNF are also known to peak to aid synaptogenesis [14, 15]. During this period, prenatal stress reduces the astrocytic pool and changes the profiles of secreted factors, which contributes to permanent defects in neurogenesis and hippocampus-related cognition [16]. Changes in astrocytic reactivity or proliferation by glucocorticoid during early life can be hypothesized to alter neurotrophic support to neurons, resulting in impaired glutamatergic or GABAergic synapse formation due to a decline in downstream factors participating in migration, proliferation, and synaptic trafficking. Therefore, we sought to identify the interactions between the altered neurotrophic factors produced by these affected astrocytes and the responsive synaptogenic regulators as putative modulators of neurogenesis under prenatal stress.

We used human induced pluripotent stem cell (iPSC)-derived NSCs, a common in vitro model for observing neurogenesis, to investigate how glucocorticoid affects synaptic plasticity and the differentiation potential of NSCs. In addition, we exposed ICR littermates to prenatal glucocorticoid injection to assess its effect on neurogenesis and subsequent behavioral deficits. Using both in vitro and in vivo models, the study addresses the effect of glucocorticoid on astrocyte-neuron interactions and the ensuing dysfunction of synaptogenesis, which results in impairments to neurogenesis or behavior.

\section{Materials And Methods}

\section{Materials}

Fetal bovine serum (\#SH30088.031R) and antibiotic-antimycotic mixture (\#15240062) were purchased from Hyclone (Logan, UT, USA) and Gibco (Grand Island, NY, USA), respectively. The antibodies of lamin A/C (\#sc-20681), pancadherin (\#sc-59876), gephyrin (\#sc-25311), and $\beta$-actin (\#sc-47778) were purchased from Santa Cruz Biotechnology (Paso Robles, CA, USA). The antibodies of MAP2 (\#ab11267), GFAP (\#ab7260), ALDH1L1 (aldehyde dehydrogenase 1 family member L1, \#ab190298), STAT3 (\#ab76318), GluR2 (\#ab133477), NR1 (\#ab109182), synaptophysin (\#ab32127), and neuroligin 1 (\#ab186279) were obtained from Abcam (Cambridge, MA, USA). The GR antibody (\#12041S) was acquired from Cell Signaling Technology, Inc. (Danvers, MA, USA). The antibodies of vGAT (\#NBP220857) and C3 (\#NBP1-32080) were purchased from Novus Biologicals (Littleton, CO, USA). The 
antibodies of DCX (doublecortin, \#PA5-17428), NeuN (\#MA5-33103), c-Fos (\#MAS-15505), vGLUT1 (\#482400), GluR1 (\#PA1-46151), NR2B (\#71-8600), FGF2 (\#OSG00015W), FGFR1 (\#MA1-26256), BrdU (\#115071-42), and PSD95 (\#51-6900) were purchased from Thermo Fisher (Rockford, IL, USA). The antibodies of a-tubulin (\#T6074) and PSD95 (\#MAB1596), cortisol (\#H4001), corticosterone (\#C2505), actinomycin D (\#A1410), AMPA (a-amino-3-hydroxy-5-methyl-4-isoxazole propionic acid, \#A6816), NMDA (N-methyl-Daspartate, \#M3262), and DAPI (\#23397) were obtained from Sigma Chemical Company (St. Louis, MO, USA). The plasmids for pcDNA3.1/FGFR1-c-eGFP, pcDNA3.1/NLGN1-c-eGFP, and pcDNA3.1/c-eGFP were purchased from KomaBiotech (Seoul, Korea).

\section{Cell culture}

The NSC induction was conducted from iPSCs obtained from Kangstem Biotech (Seoul, Korea) using neural induction medium (\#A1647801, Gibco). We plated NSCs on geltrex LDEV-Free (\#A1413302, Thermo Fisher)-coated plates. For neural differentiation, NSCs were cultured in a neurobasal medium (\#21103049, Gibco) with the 2\% serum-free supplement B27 (\#17504044, Gibco) and 1\% Glutamax (\#35050061, Gibco). For astrocytic differentiation, NSCs were incubated in high glucose DMEM (\#SH30022.01, Hyclone) containing 1\% N-2 supplement (\#17502048, Gibco), 1\% FBS, and 1\% Glutamax (\#35050-061, Gibco). After 5 days of astrocytic differentiation, astrocytes were cultured on neural differentiation media for $48 \mathrm{~h}$ with cortisol and ACM were harvested.

Cultures of mouse primary hippocampal astrocytes were performed as described following modified protocol [17]. Hippocampal astrocytes from P1 micee were acquired in compliance and approval with the Institutional Animal Care and Use Committee of Seoul National University (SNU-190523-1-1). In brief, hippocampal astrocytes cultured on poly- D $^{-l y s i n e ~ c o a t e d ~ 6-w e l l ~ p l a t e s ~ w e r e ~ i n c u b a t e d ~ i n ~ M E M ~(H y c l o n e) ~}$ supplemented with glucose $(0.6 \% \mathrm{wt} / \mathrm{vol}), 10 \%$ horse serum ( $\mathrm{vol} / \mathrm{vol})$, and $1 \%$ antibiotic-antimycotic mixture.

\section{Transfection of plasmid DNA}

Prior to ACM treatment, neurons were incubated with a mixture of plasmid DNA, opti-MEM, and lipofectamine 3000 (Thermo Fisher). Lipid-based transfection method using lipofectamin 3000 were performed in differentiating neurons following modified protocol [17]. After incubation, neurons were replaced to remove lipofectamine reagent with fresh neurobasal media containing $2 \%$ B27 supplement and $1 \%$ Glutamax.

\section{Transfection of small interfering RNA (siRNA) for gene silencing}

Neurons were grown until approximately $60 \%$ confluency of the plate. Prior to ACM treatment, differentiating neurons were incubated with mixture of $25 \mathrm{nM}$ indicated siRNAs and the turbofect transfection reagent (Thermo Fisher) for $24 \mathrm{~h}$ according to the manufacturer's instructions. The siRNAs specific for human $G R$ were purchased from Bioneer Corporation (Daejeon, Korea). The siRNAs specific for nontargeting (NT) were obtained from Dharmacon (Lafayette, CO, USA). 


\section{Reverse transcription-PCR and real-time PCR}

RNA samples were isolated using MiniBEST Universal RNA extraction kit (Takara). To acquire cDNA, reverse transcription was conducted using $1 \mu \mathrm{g}$ of RNA for $1 \mathrm{~h}$ at $45^{\circ} \mathrm{C}$, followed by 5 min at $95^{\circ} \mathrm{C}$ with a Maxime RT-PCR premix kit (Intron Biotechnology, Seongnam, Korea). Then, cDNA samples were amplified using Quanti NOVA SYBR Green PCR Kits (Qiagen, Hilden, Germany). Real-time quantification of RNA targets was performed with a Rotor-Gene 6000 real-time thermal cycling system (Corbett Research, NSW, Australia). Data were acquired during the extension step and then melting curve analysis was conducted to validate the specificity and identity of the PCR products. The data were normalized with the ACTB mRNA expression levels.

\section{RNA sequencing analysis}

For the transcriptome profiling, bilateral hippocampus from littermates of mice received vehicle or corticosterone were dissected manually at P1. For each RNA sample, the library construction was performed using the SENSE 3' mRNA-Seq Library Prep Kit (Lexogen, Vienna, Austria) according to the manufacturer's instructions. High-throughput sequencing was conducted as single-end 75 sequencing using NextSeq 500 (Illumina, San Diego, CA, USA). RNA-sequencing reads were aligned to the UCSC hg19 genome build. Scatter plots were generated on differentially expressed genes more than 2 folds between the littermates of mice with vehicle and the littermates of mice with corticosterone at pregnancy.

\section{Western blot analysis}

The samples were lysed with the appropriate buffer containing protease and phosphatase inhibitors. Cell debris was removed by centrifugation $\left(13,000 \times \mathrm{g}\right.$ at $\left.4{ }^{\circ} \mathrm{C}, 30 \mathrm{~min}\right)$. Protein determination was done by a bicinchoninic acid quantification assay (Thermo Fisher). Equal amounts of sample proteins $(1-5 \mu \mathrm{g})$ were subjected to $8-12 \%$ SDS-PAGE and transferred to a polyvinylidene fluoride membrane. Then, the membranes were blocked with $5 \%$ bovine serum albumin (Sigma Chemical Company) or $5 \%$ skim milk (Gibco) in tris-buffered saline containing 0.2\% Tween-20 \{TBST; $150 \mathrm{mM} \mathrm{NaCl}, 10 \mathrm{mM}$ Tris- $\mathrm{HCl}(\mathrm{pH} 7.6)$, $0.1 \%$ Tween-20\} solution for 30 min. After washing with TBST three times, the membranes were probed with primary antibody in $4^{\circ} \mathrm{C}$ overnight. Next, the membranes were washed three times with TBST, followed by incubation with the HRP-conjugated secondary antibodies capturing mouse or rabbit IgG (Thermo Fisher) at room temperature for $2 \mathrm{~h}$. The acquired membranes were detected with chemiluminescence solution (BioRad, Hercules, CA, USA) and the densitometry analysis for quantification was performed by using Image $\mathrm{J}$ software (developed by Wayne Rasband, National Institutes of Health, Bethesda, MD, USA).

\section{Co-immunoprecipitation}

Primary antibodies were immobilized with SureBeads ${ }^{\mathrm{TM}}$ protein $\mathrm{G}$ magnetic beads (BioRad). Immobilized magnetic beads were incubated with the total lysates of cells $(200 \mu \mathrm{g})$ at $4{ }^{\circ} \mathrm{C}$ overnight. Magnetic beads were pulled-down by magnet and then acquired. The antibody-bound protein was collected by incubation 
in elution buffer (Thermo Fisher). Protein analysis was performed by western blot analysis where antimouse or rabbit IgG antibodies were used as a negative control.

\section{Immunocytochemistry}

Cultured cells were fixed with $4 \%$ paraformaldehyde for $15 \mathrm{~min}$ at room temperature and then incubated in $0.1 \%$ tritonX for $5 \mathrm{~min}$. Cells were placed in $5 \%$ normal goat serum (NGS) in PBS for $1 \mathrm{~h}$. Next, the cells were incubated overnight at $4{ }^{\circ} \mathrm{C}$ with primary antibody dissolved in $5 \%$ NGS. After being washed with PBS, the cells were applied for $2 \mathrm{~h}$ at room temperature with Alexa Fluor ${ }^{\mathrm{TM}}$ secondary antibody (Thermo Fisher) as appropriate. Images were acquired by super-resolution radial fluctuations (SRRF) imaging system (Andor Technology, Belfast, UK). The fluorescent intensity analysis and co-localization analysis with Pearson's correlation coefficient were performed with Fiji software (developed by Wayne Rasband, National Institutes of Health, Bethesda, MD, USA).

\section{Fluorescence imaging of synaptic vesicle recycling}

For measuring recycled synaptic vesicle, neurons were loaded with FM4-64 dye (\#T3166, Thermo Fisher) and stimulated with high $\mathrm{K}^{+}$-solution following the modified protocol [18]. Images were acquired before and after destaining with high $\mathrm{K}^{+}$-solution for $90 \mathrm{sec}$ at $1 \mathrm{sec}$ intervals using Eclipse $\mathrm{Ts}^{\mathrm{TW}}{ }^{\mathrm{W}}$ fluorescence microscopy (Nikon, Tokyo, Japan) and underwent analysis of destaining kinetics.

\section{Flow cytometry}

Cells and hippocampal tissues were collected and dissociated with $1 \mathrm{X}$ Versene solution (Gibco). After being washed with PBS, cells were fixed with TF Fix/Perm buffer (\#562575, BD Bioscience, Franklin Lakes, NJ, USA) and resuspended in Perm/Wash buffer at $5 \times 10^{5}$ cells per $100 \mu$ l. The cells were incubated with primary antibody at $4{ }^{\circ} \mathrm{C}$ for $2 \mathrm{~h}$ and then applied in secondary antibody at $4{ }^{\circ} \mathrm{C}$ for $1 \mathrm{~h}$ as appropriate. After washing with PBS, the samples were detected with flow cytometry (Quanta SC; Beckman Coulter, Brea, CA, USA) and data analysis was conducted with CytExpert 2.3 software provided from Beckman Coulter.

\section{Annexin V apoptosis assay}

Annexin V-FITC/PI staining was conducted with an Annexin V-FITC apoptosis detection kit (\#BD 556547, BD Bioscience). The analysis was performed following the manufacturer's instructions. After treatment, astrocytes were suspended in binding buffer. Next, Annexin V-FITC and PI were added to the samples and incubated for $15 \mathrm{~min}$ at room temperature. Apoptosis of the samples was detected with flow cytometry (Quanta SC) and data analysis was conducted with CytExpert 2.3. Annexin V-FITC only positive cells undergo early apoptosis, whereas PI-positive only cells undergo necrosis. Both Annexin V-FITC and PIpositive cells undergo late apoptosis.

\section{Chromatin immunoprecipitation (ChIP)}


ChIP assay was conducted by using an EZ-ChIP Chromatin Immunoprecipitation Kit (\#17-371, EMD Millipore, Burlington, MA, USA) following the manufacturer's instructions. Samples including proteinchromatin complexes were incubated with ChIP grade antibody for FGFR1, the RNA polymerase (RNAPol), and normal IgG overnight at $4{ }^{\circ} \mathrm{C}$. RNAPol and normal IgG were used as a positive and negative control, respectively. Sample DNA was acquired by supplied column and amplified by PCR using a designed primer. The sequences of NLGN1 primer are as follows: forward primer, 5'-

TGAAGCAAGCTCTTAAATGGTG-3' and reverse primer, 5'-TAATCGTAACCCCAAAAGGAAA-3'. The sequences of NLGN3 primer are as follows: forward primer, 5'-TGCTAAACAAATGGCAGGTG-3' and reverse primer, 5'-ACCTCCACCTCAATCAGCAT-3'. The sequences of NRXN2 primer are as follows: forward primer, 5'-CCAGCACCACTCTAACTGAAAC-3' and reverse primer, 5'-GCTTTGCTGGGATAAAGACG-3'. The sequences of NRXN3 primer are as follows: forward primer, 5'-TGCCTCAAGGGGTTTATTTTTA-3' and reverse primer, 5'-ACACTGCGCCTTCTTTATCAC-3'. One percent of the sample chromatin extract was used as an input.

\section{Experimental design of animal study}

Female pregnant ICR mice exposed to corticosterone mimic the maternal stress-induced mouse model since corticosterone is primarily responsive steroid hormone to stress. Then, the hippocampus of littermates was mainly used for evaluating glucocorticoid effect on neurogenesis since the hippocampus is closely related to neuropsychiatric and cognitive function among the brain. Female pregnant ICR mice aged 8 weeks were used, in compliance and approval with the Institutional Animal Care and Use Committee of Seoul National University (SNU-200221-6-1). Both sexes of ICR mice aged 8 weeks were also used to compare the effect of stress between infant and adult mice, in compliance and approval with the Institutional Animal Care and Use Committee of Seoul National University (SNU-190917-6-1). Animals were kept under standard environmental conditions $\left(22^{\circ} \mathrm{C}\right.$ relative humidity $70 \% ; 12 \mathrm{~h}$ light: dark cycle; ad libitum access to food and drinking solution). At least six mice were utilized for each group throughout the study. Applying size of samples (minimum of $n=3$ ) can be acceptable if very low $p$ values are observed rather than the large size of $\mathrm{N}$ including interfering results. Therefore, we set the minimum of $n=3$ (western blotting, immunohistochemistry) and $n=5$ (behavior test) to gain statistical powers according to the previous published article of Brain. The experiments were designed in compliance with the ARRIVE guidelines. Allocations of animals were randomly done to minimize the effects of subjective bias.

Corticosterone $(10 \mathrm{mg} / \mathrm{kg}$ ) was dissolved in the solution containing $50 \%$ propylene glycol in PBS and injected intraperitoneally, which induces maternal stress in female pregnant mice and adult stress in 8week-old mice $[13,19]$. Vehicle-treated mice were similarly injected with the solution containing propylene and PBS. FGF2 (20 ng/g) was dissolved in the PBS. The dosage and treatment period of FGF2 is modified from previous reports $[14,20]$. FGF2 was injected subcutaneously into axillary space. Mice were monitored twice a day during all experiments.

\section{Y-maze spontaneous alternation test}


Y-maze spontaneous alternation test depends on the innate instinct of rodents to differently explore new environments. Thus, this test is widely used for quantifying the spatial memory of rodents. Rodents often prefer to challenge a new arm of the Y-maze rather than returning back to the one previously explored. Before the performance, the animals were habituated to the testing room for $3 \mathrm{~h}$ to minimize the stress. Then, mice were allowed for 10 min to explore the Y-shaped maze purchased from Sam-Jung Company (Seoul, Korea) while the number of arm entries and triads were recorded to calculate the percentage of alternation. Only an entry when all four limbs were within the arm was counted. The alternation value represents the number of alternations which was divided by total triads (total entries-2).

\section{Forced swim test}

Forced swim test is a behavior test for rodents to evaluate the depressive-like behavior. Before the performance, the animals were habituated to the testing room for $3 \mathrm{~h}$ to minimize the stress. Mice were subjected to a forced swim test for $6 \mathrm{~min}$ in a beaker $(10 \mathrm{~cm} \times 20 \mathrm{~cm})$ filled with tap water at room temperature, and the trials were analyzed by Smart 3.0 video tracking system (developed by Panlab, Barcelona, Spain). Generally, only the last 4 min of the test is analyzed due to the fact that most mice rigorously try to escape the environment at the beginning of the test. Immobility was defined as floating or remaining motionless without leaning against the wall of the cylinder.

\section{Open field test}

Open field test evaluates anxiety of rodents using their characteristics to explore periphery of open field when anxious. Before the performance, the animals were habituated to the testing room for $3 \mathrm{~h}$ to minimize the stress. Mice were placed in the rectangular plastic black boxes $(\mathrm{H} 30 \times \mathrm{L} 30 \times \mathrm{W} 30 \mathrm{~cm})$ and activity was recorded for $10 \mathrm{~min}$. Total distance explored and time spent in either center or periphery of the open field were analyzed using Smart 3.0 video tracking system.

\section{Dexamethasone suppression test and corticosterone assay}

Before the test, the blood was acquired from tail vein. For dexamethasone suppression test, mice were injected with dexamethasone ( $50 \mu \mathrm{l} / \mathrm{kg}$ in propylene glycol) $1 \mathrm{~h}$ before acquiring blood from the abdominal aorta of mice just before sacrifice. Then, plasma was separated from whole blood by centrifugation. To analyze, concentration of plasma corticosterone was measured using the corticosterone ELISA kit purchased from Enzo Life Science (Farmingdale, NY, USA), according to the manufacturer's instructions.

\section{Synaptosome isolation}

Synaptosome of mouse hippocampus and neurons from human iPSC-derived NSC was extracted using Syn-Per synaptic protein extraction reagent (Thermo Fisher). Hippocampus and neurons were homogenized with Dounce grinder with 20 slow strokes. Then, the homogenates underwent centrifugation at $1,200 \times \mathrm{g}$ for $10 \mathrm{~min}$ at $4{ }^{\circ} \mathrm{C}$. After discarding the pellet, the supernatant was centrifuged 
at $15,000 \times \mathrm{g}$ for $20 \mathrm{~min}$ at $4^{\circ} \mathrm{C}$. The supernatant and pellet represent the cytosol and synaptosome, respectively.

\section{Growth factor antibody array}

The conditioned media extracted from astrocytes were analyzed for their content of growth factors by incubation with membranes of the RayBiotech C-Series Human Growth Factor Antibody Array C1 kit, AAHGF-1 (RayBiotech, Norcross, GA, USA). The membranes were incubated in blocking buffer for 30 min and then placed with conditioned media overnight at $4{ }^{\circ} \mathrm{C}$. Membranes were then washed five times with wash buffer and incubated with biotin-conjugated antibodies for $2 \mathrm{~h}$ at room temperature. After five times of wash with wash buffer, the membranes were incubated with horseradish peroxidase-conjugated streptavidin for $2 \mathrm{~h}$. After washing process, the human growth factors were detected by enhanced chemiluminescence reagents using a chemiluminescence imaging system.

\section{ELISA for human and mouse FGF2}

For the quantification of FGF2 in conditioned media or tissue lysates, the FGF2 ELISA assay was achieved with human FGF2 ELISA kit (abcam, \#ab246531) and mouse FGF2 ELISA kit (abcam, \#ab100670) according to supplier's protocols.

\section{Immunohistochemistry (IHC)}

Mice were subjected to deep anesthesia with zoletil (50 mg/ $\mathrm{kg}$ ) and perfused transcardially with calciumfree Tyrode's solution, followed by $4 \%$ paraformaldehyde. The brains were post-fixed for $2 \mathrm{~h}$ in $4 \%$ paraformaldehyde and then dehydrated in $30 \%$ sucrose in PBS for $24 \mathrm{~h}$ at $4{ }^{\circ} \mathrm{C}$. Serial transverse sections $(40 \mu \mathrm{m})$ were conducted using a cryostat (Leica Biosystems, Nussloch, Germany). The brain tissues including dorsal or ventral hippocampus were fixed with $4 \%$ paraformaldehyde for $10 \mathrm{~min}$, and then preblocked with $5 \%$ NGS in PBS at room temperature for $1 \mathrm{~h}$. Brain samples were incubated with primary antibody overnight at $4{ }^{\circ} \mathrm{C}$, followed by secondary antibody for $2 \mathrm{~h}$ at room temperature as appropriate. All completed samples were visualized using SRRF imaging system. The fluorescent intensity analysis and determining Pearson's correlation coefficient values were undertaken using Fiji software.

\section{Statistical analysis}

Imaging experiments and animal tests were conducted and assessed in a blinded-fashion. Sample sizes were kept similar between experimental groups and replicates of experiments. The sample size ' $n$ ' represents the number of biological independent replicates and statistical analyses were conducted using these independent values. The unpaired student's t-test was performed to compare the means of the treatment groups with that of the control group. One-way ANOVA (with Dunnett's multiple comparison test) or two-way ANOVA (with Tukey's multiple comparison test) were used for analyzing the differences among multiple groups. For measuring co-localization levels in images, the values of Pearson's correlation coefficient were obtained from images of each treatment group, and appropriate tests were applied to confirm whether changes were statistically significant. Results are expressed as mean value \pm 
standard error of mean (S.E.M.) and analyzed with the GraphPad Prism 6 software (Graphpad, CA, USA). A result with a $p$ value of $<0.05$ was considered statistically significant.

\section{Results}

\section{Maternal stress induces neuropsychiatric and spatial memory disorders in littermates by impairing neurogenesis}

We focused on the effect of stress on the hippocampus because of its critical role in processing emotionally salient information or regulating memory function. Stress exposure releases glucocorticoid hormones, namely cortisol in humans and corticosterone in rodents. Previous studies have noted that corticosterone exposure in fetal rodents triggers mood disorders or memory dysfunction. Thus, we used about 3-week-old mice model exposed to maternal corticosterone at embryonic day 14 (E14) to study the relationship between neurogenesis and emotional/memory behavior. Using an open field test to study anxiety behavior, in which mice with anxiety prefer to stay at the periphery, we found that those exposed to prenatal corticosterone were more active in the peripheral regions than in the center of the open field (Figure 1a). To evaluate depression-like behavior, we performed a forced swim test, which determines immobility in a cylinder, an indicator of unwillingness to escape the environment. Mice exposed to prenatal corticosterone exhibited immobility more than the control mice (Figure 1b). Furthermore, we assessed spatial memory function using the $\mathrm{Y}$-maze, which uses the innate nature of rodents to explore the new objects, and observed that mice exposed to prenatal corticosterone exhibited dysfunctional spatial memory (Figure 1c). Overall, maternal exposure to high levels of corticosterone influenced both neuropsychiatric and cognitive function in littermates. In adults, stress adaptation is usually well controlled by the hypothalamus-pituitary-adrenal (HPA) axis; however, exposure to stress during early life is known to permanently impair this axis. We validated the hyperactivation of the HPA axis due to an impaired feedback mechanism using a dexamethasone suppression test during the dark cycle. Mice were treated with dexamethasone to suppress corticosterone release $1 \mathrm{~h}$ before serum acquisition.

Dexamethasone effectively suppressed plasma corticosterone release in control mice, whereas mice exposed to prenatal corticosterone were more insensitive to the drug's suppressive effects on the HPA axis than the vehicle-treated group (Figure 1d). The effect of prenatal stress on neurogenesis was determined 28 days after initial exposure to prenatal corticosterone (postnatal day 23, P23), when the adult mice usually showed recovery. The dorsal and ventral hippocampus are widely known to be responsible for processing cognition and emotion, respectively. Thus, we performed immunostaining in both regions and detected changes in neurogenesis, especially in the SGZ, where most hippocampal neurogenesis occurs [13]. First, we investigated the effect of corticosterone on neuronal activation. An immediate-early gene $c$-fos is activated after action potential firing, and this is used to evaluate activated neurons [21]. The ratio of c-Fos-immunoreactive to surviving neurons binding to BrdU was decreased in the SGZ, suggesting that neural activity was reduced in both the dorsal and ventral hippocampus (Figure 1e). Next, we determined neuronal maturation by staining neural tissue with DCX and NeuN, which identify immature and terminally differentiated neurons, respectively. Corticosterone exposure resulted in the increased ratio of DCX to NeuN fluorescence intensities in both the dorsal and ventral hippocampus, 
indicating that corticosterone suppressed neurogenesis (Figure 1f). Together, these results demonstrated that exposure to prenatal corticosterone adversely affected neurogenesis which can impair $\mathrm{mood} /$ memory function.

\section{A1-like astrocytes induced by glucocorticoid suppress glutamatergic synaptogenesis}

Mice exposed to stress during adulthood are reported not to exhibit behavioral disorders 5 days after withdrawal of the stress [9]. However, we suggested that the effect of maternal stress on behavior deficits persists even 4 weeks after glucocorticoid injection. Thus, we investigated whether changes to differentiation induced by glucocorticoid early in neurogenesis precede behavioral disorders. We injected corticosterone into pregnant mice at E14, when neuronal development and differentiation peak. Neurogenesis was then evaluated by flow cytometry 5 days after the injection (P1), when neurogenesis can be phenotyped by stimulation. To assess whether corticosterone modified neuronal activity and neurogenesis, we used immunostaining in the cells dissociated from the hippocampus at P1. Prenatal corticosterone injection reduced the ratio of c-Fos to BrdU immunoreactive cells (Figure 2a). Next, we observed whether corticosterone exposure at E14 increased the ratio of DCX-positive to NeuN-positive neurons (Figure $2 b$ ). These data clearly demonstrated that corticosterone depleted the neurogenic potential of NPCs.

Growing evidence indicates that astrocytes can strongly regulate NSC dynamics during the fetal or infant stage through their secreted neurotrophic factors by enhancing angiogenic function, synapse formation, and the differentiation potential of neurons, motivating us to examine whether the astrocytic pool changes in response to glucocorticoid treatment. We treated cells with $1 \mu \mathrm{M}$ cortisol or corticosterone, according to the stress-induced levels of glucocorticoid used in our previous research [19]. Approximately $20 \%$ more apoptosis occurred in astrocytes that had differentiated from NSCs exposed to cortisol for 5 days than in the control astrocytes (Figure 2c). These results concur with previous research demonstrating that glucocorticoid introduced at the fetal stage inhibits astrocyte proliferation and subsequent neurotrophic support, resulting in reduced synaptogenesis and neural development; however, contradictions to this suggestion also exist [12]. Astrocytes show heterogeneity in their reactions to various stimuli; a shift to A1-like reactive, neuroinflammatory astrocytes, which lose their neurotrophic capacity, is induced in certain brain disorders or following damage [22]. In contrast, A2-like reactive, protective astrocytes aid neuronal growth and synapse repair to maintain brain homeostasis following stimulations such as ischemic injury [23-25]. In determining whether cortisol shifts astrocytes into reactive, pathological phenotypes, we found that cortisol triggered reactive astrocytosis, resulting in elevated level of the reactive astrocyte marker GFAP compared with the nuclei marker PI (Figure 2d). Furthermore, cortisol increased the surface area of astrocytes, suggesting that they became reactive (Figure 2e). We then distinguished which types of reactive astrocytes were dominant following exposure to cortisol. Western blotting revealed that cortisol elevated the ratio of the pan-reactive astrocytic marker GFAP to the astrocyte-specific marker ALDH1L1 (Figure 2f). Simultaneously, cortisol decreased STAT3 but elevated $\mathrm{C} 3$ expression, which are the representative proteins for protective A2-like and neurotoxic A1like astrocytic markers, respectively $[25,26]$. We also confirmed that corticosterone elevated the ratio of 
GFAP to ALDH1L1 and C3 levels, but decreased STAT3 levels in mouse primary hippocampal astrocytes (Supplementary Figure 1). To observe the deleterious effects of A1-like astrocytes on neurogenesis, we extracted astrocyte conditioned media (ACM) treated with cortisol and administered them to differentiating neurons for 5 days. Cortisol-treated ACM reduced the neuronal activity and maturation of neurons (Figures $2 g-2 h$ ). Collectively, these findings suggested that glucocorticoid critically depleted the neurogenic potential of NSCs by transforming astrocytes into A1-like reactive astrocytes which may not release neurotrophic factors.

Reduced neurotrophic aid from astrocytes often culminates in less neuronal excitability, which usually precedes neurogenesis defects, due to the role of neurotrophic factors in glutamatergic or GABAergic synapse formation [27]. Thus, we measured the kinetics of FM4-64 destaining to validate the adverse effect of cortisol-treated ACM on the synaptic current in neurons [18]. When administered to differentiating neurons for $48 \mathrm{~h}$, cortisol-treated ACM reduced the rate of FM4-64 dye release, indicating that synaptic vesicle recycling and synaptic current activity were reduced (Figure $3 a$ ). To differentiate which types of synapse formation were prevented by cortisol-treated ACM, we stained the neurons with excitatory and inhibitory synaptic markers. As seen in Figure 3b, cortisol-treated ACM downregulated excitatory synapse formation (colocalization between presynaptic protein vGLUT1 and postsynaptic protein PSD95) but did not induce a significant change in inhibitory synapse formation (colocalization between presynaptic protein vGAT and postsynaptic protein Gephyrin). Furthermore, to determine which glutamatergic pathway was suppressed, synaptosomes were extracted from P1 mice exposed to corticosterone at E14. As shown in Figure 3c, corticosterone reduced the levels of AMPA receptor (AMPAR) subtypes GluR1/2 in the synaptosome of the hippocampus whereas the NMDA receptor (NMDAR) subtypes NR1 and NR2B exhibited no significant level changes in each compartment. In line with the in vivo results, cortisol-treated ACM reduced the recruitment of GluR1/2 into synaptic membrane, whereas no significant transport of NR1 and NR2B was detected in the immunofluorescence results (Figure 3d). Furthermore, cortisol-treated ACM reduced synaptic trafficking of GluR1/2 in human differentiating neurons, whereas their expression remained unchanged (Figure $3 e$ ). To confirm whether AMPA-mediated glutamatergic transmission in neurons was impaired by cortisol-treated ACM, we showed that synaptic vesicle recycling, as demonstrated by FM4-64 dye kinetics, was recovered by AMPA treatment, not by NMDA treatment (Figure 3f). Furthermore, using Sholl analysis, the number of intersections was reduced by cortisol-treated ACM, meaning that neurite outgrowth and subsequent synaptogenesis were impaired (Figure $3 \mathrm{~g}$ ). These data suggested that astrocytic phenotype changes caused by glucocorticoid highly affect AMPAR-but not NMDAR-mediated glutamatergic synaptogenesis of neurons [28].

\section{Glucocorticoid impairs communication between astrocytic FGF2 and neuronal FGFR1}

Based on the observation that cortisol-treated ACM impaired synaptic trafficking of GluR1/2, we performed RNA sequencing to determine which neurotrophic factor-dependent signaling pathway represses glutamatergic transmission during neurogenesis in prenatal stress-induced mice. We compared the gene expression profiles of control mice and those exposed to prenatal corticosterone at E14. Scatter 
plots revealed that the mRNA expression of several neurotrophic factor-associated genes including Fgfr1, Fgfr3, and Camk2a was reduced more than 2-fold following corticosterone treatment (Figure 4a). Given that astrocytes secrete a variety of growth factors in support of neurogenesis, we determined the levels of neurotrophic factors released by astrocytes. In a growth factor antibody array, cortisol particularly decreased astrocytic fibroblast growth factors (Figure $4 \mathrm{~b}$ ). These results led us to assume that FGF2 is key to maintaining synaptogenesis and subsequent neurogenesis during early life in mice since FGFR is highly associated with FGF2. FGF2 levels were decreased in extracted media from astrocytes (DIV 7) under cortisol treatment (Figure 4c). Consistently, FGF2 levels in both mouse hippocampal tissue exposed to prenatal corticosterone and media from mouse primary hippocampal astrocytes (DIV 7) were reduced (Supplementary Figures $2 a-2 b$ ). However, the FGF2 levels released from human differentiating neurons (DIV 7) were not significantly changed by cortisol, indicating that astrocytes play an important role in FGF2 support (Supplementary Figure 2c). Furthermore, FGF2 levels in fully differentiated astrocytes (DIV 21) following $48 \mathrm{~h}$ of cortisol treatment were not significantly changed (Supplementary Figure 2d). These results suggested that early-stage astrocytes play a key role in FGF2 release. By investigating whether pretreatment with human recombinant FGF2 rescues the detrimental effects of cortisol-treated ACM on neurogenesis, we found that this recovered dysregulated neurogenesis by restoring the ratio of DCXpositive to NeuN-positive cells (Figure 4d). As shown in Figure 4a, FGFR1-mediated signaling may be suppressed by astrocytic FGF2 reduction. FGFR1s are localized either in the cytosol, nucleus, or membrane, each of which has a distinct signaling pathway [29]. To differentiate which signaling pathway is activated, we performed subcellular fraction and immunostaining to find that cortisol-treated ACM selectively decreased the levels of nuclear FGFR1, which were recovered by FGF2 pretreatment (Figure 4e). Similarly, the immunostaining results showed that the amount of FGF2-FGFR1 complex in the nucleus was reduced by cortisol-treated ACM, and this was reversed by FGF2 pretreatment (Figure 4f). FGF2 is classified into high molecular weight (HMW $22-25 \mathrm{kDa}$ ) and low molecular weight (LMW 18 $\mathrm{kDa}$ ) isoforms. FGFR1 bound to LMW FGF2 at the membrane usually acts as a receptor tyrosine kinase (RTK) and normally activates other kinases responsible for mitogenic function. As it contains the nuclear localization sequence, HMW FGF2 and its receptor FGFR1 were transported into the nucleus. When trafficking into nucleus, large parts of full-length FGFR1 $(120 \mathrm{kDa})$ become truncated $(55-60 \mathrm{kDa})$ and both forms bound to HMW FGF2 normally promote neural differentiation by binding to DNA [29, 30]. This pathway is called integrative nuclear FGFR1 signaling (INFS), because nuclear FGFR1 cooperates with the CREB binding protein and many other transcription factors such as RXR and CREB [31]. To confirm whether the interaction between HMW FGF2 and INFS was selectively affected, we performed immunoprecipitation assays of nuclear parts, which demonstrated that cortisol-treated ACM selectively suppressed nuclear FGFR1 binding to HMW FGF2 rather than LMW FGF2, indicating that neuronal INFS was depressed by cortisol-treated ACM (Figure 4g).

We then evaluated whether nuclear FGFR1 mainly participates in regulating glutamatergic synapse formation. We neutralized membrane FGFR1 with FGFR1 antibody to determine whether the adverse effects of cortisol-treated ACM depend on lower levels of nuclear FGFR1 in neurons. Trafficking of GluR1/2 into the postsynaptic compartment in differentiating neurons was blocked by cortisol-treated 
ACM but overexpression of FGFR1 attenuated these effects. However, FGFR1 antibody did not reverse these recovery effects, suggesting that nuclear FGFR1-mediated signaling plays an important role in maintaining the AMPA-mediated glutamatergic pathway (Figures $5 a-5 b$ ). Furthermore, synaptic vesicle recycling recovered by FGFR1 upregulation was not blocked by the FGFR1 antibody (Figure 5c). We also detected dendritic spine density to determine the amount of synaptogenesis. As shown in Figure $5 \mathrm{~d}$, reduced neurite outgrowth caused by cortisol-treated ACM was recovered by overexpression of FGFR1, which remained unchanged by the addition of FGFR1 antibody. Altogether, decreased astrocytic FGF2 by glucocorticoid triggered defects in glutamatergic synaptogenesis, which was mainly dependent on a suppressed INFS.

\section{Neuroligin 1 downregulation by glucocorticoid reduced glutamatergic pathway-dependent neurogenesis and triggered behavioral deficits}

Nuclear FGFR1 bound to HMW FGF2 attaches to DNA and acts as a transcription factor for various neurogenesis and neural activity-associated genes. Because changes in GluR1/2 expression are not detected in our results, we assumed that synaptic GluR1/2 were selectively reduced by suppressed INFS. To maintain the synaptic pools of GluR1/2, transporting or scaffolding GluR1/2 into the postsynaptic compartment is critical to glutamatergic synapse formation and subsequent long-term potentiation (LTP). Thus, we observed mRNA expression of MYO5, which transports GluR1/2 into the synapse, and synaptic cell adhesion-associated genes, including NLGN isoforms and NRXN isoforms. The genes for neuroligin $1 / 3$, neurexin $2 \alpha / \beta$, and neurexin $3 \beta$ were downregulated by cortisol-treated ACM (Figure 6a). Nuclear FGFR1-targeted motifs are known to include a core AGGTCA sequence and we searched for this putative region for FGFR1 binding to the promoters of downregulated genes [30]. Thus, we performed in silico assay and designed the primers referring to the results (Supplementary Figures $3 a-3 d$ ). Then, ChIP was performed to determine the genes most highly regulated by nuclear FGFR1. As shown in Figure 6b, cortisol-treated ACM only reduced the binding of FGFR1 to the NLGN1 promoter, while binding to the NRXN2/NLGN3 promoter was not observed and that to the NRXN3 promoter remained the same. Overexpression of FGFR 1 also recovered neuroligin 1 expression, but the transcription inhibitor actinomycin $D$ did not reverse the effect of cortisol-treated ACM on neuroligin 1 expression, indicating that INFS plays a pivotal role in neuroligin 1 transcription (Figure 6c). Furthermore, overexpression of NLGN1 recovered GluR1/2 trafficking into the postsynaptic compartment, synaptic vesicle recycling, neurite outgrowth, and neurogenesis (Figures $6 \mathrm{~d}-6 \mathrm{~g}$ ). We tested whether neuroligin 1 was altered in fully differentiated neurons to confirm that cortisol-treated ACM critically affects early neurogenesis of NSCs. Neuroligin 1 levels in fully differentiated neurons (DIV 21) did not change following exposure to cortisol-treated ACM, unlike those in differentiating neurons (DIV 7) (Figure 6h). Furthermore, we even tested the effect of GR-mediated signaling in neuroligin 1 expression. Since GR expression can vary depending on the stages of neuronal differentiation, we tried to demonstrate that neuroligin 1 expression is highly associated with other signaling pathways independent from GR at the early stage. Knockdown of $G R$ did not further decrease neuroligin 1 expression suppressed by cortisol-treated ACM (Supplementary Figure 4). Collectively, these results demonstrated that neuroligin 1 downregulation 
caused by reduced INFS following exposure to cortisol plays an important role in glutamatergic synapse formation and subsequent neurogenesis, especially in the early stage of differentiating NSCs.

With the pathway having been established, we performed in vivo experiments to explore corticosteroneinduced dysfunctional brain phenotypes. To confirm the significant role of prenatal glucocorticoid on neuroligin 1 expression, we administered corticosterone to mice at E14 and 8-week-old mice and compared the neuroligin 1 expression between the two groups. Similar to the in vitro results, fetal corticosterone exposure downregulated neuroligin 1, but no significant change was seen in the adults (Figure 7a). To assess glutamatergic synapse formation, we stained brain tissue with vGLUT1 and PSD95. Pretreatment with mouse FGF2 increased the interaction between vGLUT1 and PSD95, which was reduced by prenatal glucocorticoid exposure (Supplementary Figure 5a). Furthermore, pretreatment with FGF2 normalized colocalization between GluR1/2 and PSD95, demonstrating that it attenuated the inhibitory effect of corticosterone on the postsynaptic trafficking of GluR1/2 (Figure 7b). We also performed synaptosome isolation and observed that synaptic trafficking of GluR1/2 was recovered by FGF2 pretreatment despite corticosterone exposure (Figure 7c). Consistently, an immunoprecipitation assay demonstrated that the reduced localization of GluR1/2 and neuroligin 1 in postsynaptic compartments due to corticosterone treatment was restored by FGF2 pretreatment (Figure 7d). Moreover, we showed that the reduction in nuclear FGFR1 caused by prenatal glucocorticoid exposure was reversed by FGF2 pretreatment (Supplementary Figure 5b). Decreased glutamatergic synaptogenesis and neurogenesis in the hippocampus eventually culminates in behavioral changes such as anxiety-like behavior, major depressive disorder, and spatial memory dysfunction. We confirmed that FGF2 pretreatment drove mice to explore the center of the open field rather than the periphery (Figure 7e). As shown in the results of forced swim test, FGF2 pretreatment rescued the depressive mood induced by corticosterone exposure (Figure 7f). We also observed from the $\mathrm{Y}$-maze alternation test results that corticosterone-treated mice exhibited impaired spatial memory, but pretreatment with FGF2 attenuated the damage to cognitive function (Figure $7 \mathrm{~g}$ ). Altogether, neuroligin 1-dependent glutamatergic synapse formation and neurogenesis are selectively inhibited following prenatal exposure to corticosterone due to the suppression of the astrocytic FGF2-neuronal INFS axis.

\section{Discussion}

This study provides evidence that prenatal glucocorticoid exposure reduces neuroligin 1-dependent GluR1/2 trafficking into the synapse and subsequent neurogenesis in mouse hippocampus/human iPSCderived NSCs, and this decrease is induced by the downregulation of astrocytic FGF2 and the downstream nuclear FGFR1 in neurons (Figure 8). Our results and those from previous studies demonstrate that prenatal exposure to high levels of glucocorticoid affect both dorsal and ventral hippocampal neurogenesis, while this effect is not seen in infants or adults exposed to stress [2]. Sensitivity to stress is disputed to differ between the dorsal and ventral hippocampus. For example, insignificant changes in spatial memory function in rodents exposed to early stress were observed although contradicting results have been found depending on the early-life stress protocols [32, 33]. In contrast, our previous research demonstrated that only spatial memory dysfunction was detected while 
depressive behavior was not observed in adult mice following 1 week of exposure to corticosterone [19]. We speculated that these unique characteristics induced by prenatal stress may be due to different epigenetic and transcriptome profiles between the two regions, which can create a discrepancy in the onset of spatial memory and mood disorders depending on the time of exposure to corticosterone $[4,10$, $32,34]$. Furthermore, changes in GR levels during fetal exposure to prenatal stress can trigger unique cognitive or mood responses. Besides the fact that GR expression differs depending on the stage of neuronal differentiation, our results and previous studies have shown that prenatal corticosterone impairs the HPA axis in P23 mice, meaning that a reduction in GR would exaggerate HPA axis at this age [35]. Thus, other glucocorticoid-mediated crosstalk pathways independent from GR-mediated signaling such as changes in neurotrophic factors released from glial cells by prenatal glucocorticoid can largely affect differentiation in immature neurons [2, 8]. Finally, we presumed that the distinct characteristics of hippocampal neurogenesis during the prenatal period also contribute to the deleterious effect of glucocorticoid on neural differentiation. Prenatal stress drives NSC differentiation into glial cells, as in oligodendrogenesis, causing an inability to buffer stress due to insufficient pools of hippocampal NSCs or neurons $[2,7,16]$. The size of the NSC pool at the SGZ when responding to stress also contributes to hippocampal neurogenesis, because relatively large portions of infant NSCs contribute to brain circuit formation, unlike adult NSCs which are usually differentiated to buffer stress. Collectively, it is reasonable to conclude that prenatal glucocorticoid predisposes animals and humans to develop cognitive or neuropsychiatric disorders through distinct mechanisms that negatively affect neurogenesis, unlike earlylife or adult stress.

We have demonstrated that prenatal glucocorticoid promotes astrocytes to become A1-like reactive astrocytes, which are toxic to synaptogenesis and neurogenesis due to their inability to release neurotrophic factors. We thereby investigated the research regarding the effect of ACM on synapse formation of NSC and found that cortisol-treated ACM only affected AMPA-mediated glutamatergic synapse formation. GABAergic and glutamatergic neurons represent two major neuronal classes, which establish inhibitory and excitatory synapses, respectively. In the SGZ, GABAergic interneurons regulate synaptic integration in early neurogenesis, while glutamatergic mechanisms maintain neuronal plasticity at later stages in neurogenesis [36,37]. Our results and previous work suggest that prenatal stress dominantly influences the later stage of neurogenesis, which agrees with the finding that the migration of GABAergic interneurons does not require certain neurotrophic factors such as FGF2 [38]. We then determined why cortisol-treated ACM selectively amplifies the defects in AMPA-mediated glutamatergic synaptogenesis. To activate glutamatergic synapse formation, the ratio of GluR1/2 to NR in the synapse increases, because GluR subunits are major contributors to LTP formation. And incorporation of AMPAR into the excitatory synapse later activates NMDAR, which can form the late phase of LTP even weeks after the stimuli [1, 39-41]. In our research, prenatal stress selectively decreased AMPA-mediated synaptic transmission, but only the synaptic trafficking of GluR subunits was suppressed, unlike the downregulation of GluR1 and NR1 shown in neurons of the adult rats given 4 weeks of repeated stress [9]. High levels of glucocorticoid are widely known to ultimately induce upregulations of NMDAR levels due to the elevated endocytosis of GluR subunits [28, 41, 42]. Thus, there is a possibility that longer term 
observations of LTP formation following prenatal stress would offer different results from our results; however, we presumed that discrepancies can occur due to the different neurotrophic factors that contribute to glutamatergic synapse formation depending on the period of exposure to glucocorticoid.

Our transcriptomic approach and comparison depending on the timing of exposure to glucocorticoid revealed that astrocytic FGF2 release was significantly reduced in the hippocampus under prenatal stress and in differentiating astrocytes from human NSCs under cortisol treatment, unlike stress-exposed adult mice or fully differentiated astrocytes. In fetuses or neonates, profiles of and sensitivity to neurotrophic factors are different from those in adulthood. For example, FGF2 is abundant in embryonic brains compared to other neurotrophic factors and peaks at E14 - E18 in rodents. It is maintained at a high level until the early-postnatal stage to conserve the astrocytic and neuronal pool, but significantly decreases during mid-to-late neurogenesis $[15,38]$. Thus, excessively low levels of FGF2 inhibit the proliferation and differentiation of astrocytes, so that NSC pools remain undifferentiated, decreasing neurogenesis and gliosis in early life [20,33]. In contrast, FGF4, which is deficient in neonatal precursor cells, dominates during the later stages [14]. Other isotypes such as FGF3, FGF7, FGF8, FGF15, FGF17, and FGF18 regulate neurogenesis in specific regions, whereas FGF2 affects both neurogenesis and gliosis throughout the brain [43]. These findings suggest that the time-specific effects of FGF2 dominantly and selectively occur during early NPC activity, when they are susceptible to prenatal stress. Interestingly, the levels of FGF2 released from differentiated neurons did not change significantly, which is consistent with previous research demonstrating that astrocytes are the dominant FGF2-producing cells in the CNS [44]. Collectively, it can be inferred that a decreased astrocytic pool and an elevated number of neurotoxic astrocytes caused by prenatal glucocorticoid worsen neurogenesis by reducing fetal or neonatal FGF2 release.

FGF2 is widely known to affect neurogenesis through its receptor FGFR. FGFR isotypes distribute differentially in the brain; FGFR1/4 are predominant in neurons whereas FGFR2/3 mainly exist in oligodendrocytes and astrocytes [20]. According to previous research, FGFR1 is highly expressed in NSCs and the FGF2-FGFR1 system is especially critical to CNS formation during early life, whereas FGFR4 is not essential to embryonic brain development [38]. Consistently, our results demonstrated that the decreased interaction between astrocytic FGF2 and neuronal FGFR1 due to prenatal glucocorticoid exposure is mostly responsible for suppressing glutamatergic synaptic transmission in NSCs. Importantly, however, our results are not consistent with the previous paradigm that the FGF2-FGFR1 system mainly activates the RTK action of FGFR1 by phosphorylating tyrosine 766 , subsequently triggering downstream candidates such as PI3K, RAS-MAPK, and PKC [39, 45]. Previous research demonstrated that AMPAR-mediated synaptic transmission in neurons depends on the LMW FGF2membrane FGFR1-PKC axis [20]. But it turned out that the interaction between HMW FGF2 and INFS is a key mediator of impaired glutamatergic synapse formation and the subsequent neurogenesis of NSCs, independently from RTK action triggered by surface FGFR1-mediated signaling. We must then ask why prenatal glucocorticoid exposure primarily affects nuclear FGFR1-dependent transcription in neurons. As mentioned above, signal transduction associated with neurotrophic factor-mediated neuronal differentiation occurs differentially in the various brain regions. For example, FGFR1 preferentially affects 
the ventral hippocampus, suggesting that the FGF2-FGFR1 system can be differentially activated [13]. Moreover, the different roles of FGFR1 which is present in intracellular compartments, or release of FGF2 isotypes can be changed depending on the type of stimuli [29]. FGFR1 is usually a membrane-directed protein, however, with HMW FGF2 stimulation, FGFR1 is released from the pre-Golgi membrane into the cytosol by Sec61 and the binding of ligands can change the number of FGFR1 molecules in each pool [29]. Furthermore, INFS is known to affect brain development and differentiation, unlike RTK action by membrane FGFR1 usually contributes to mitogenic function [30]. Thus, decreased HMW FGF2 release from astrocytes exposed to prenatal glucocorticoid would selectively suppress FGFR1 transcription. Another possibility we can also assume is that persistent changes in the epigenetic profiles derived from altered neurotrophic factors by prenatal stress can affect the number of FGFR1s in each compartment and increase the nuclear pool facilitating DNA transcription in neurons. The expression of molecules such as importin and notch that participate in FGFR1 translocation into the nucleus could be also differentially expressed, depending on the brain region or glucocorticoid exposure period [46]. Even though these speculations require further investigation, this is the first observation about the unique suppressive effects of prenatal glucocorticoid exposure glutamatergic synapse formation and neurogenesis by impairing the interaction between astrocytic FGF2 and neuronal INFS.

An important finding of this study is that decreased INFS caused by prenatal glucocorticoid exposure downregulated neuroligin 1, a critical mediator of glutamatergic synapse formation. Glutamate receptormediated synaptic transmission is mainly induced by an increased surface ratio of AMPAR/NMDAR, and the activation of an AMPA-mediated synaptic current subsequently activates various factors such as CaMKII, PKC, PKA, ERK, and CREB which aid neurogenesis and memory formation [28]. Among the genes participating in trafficking or scaffolding of GluR1/2 into synapse, we found that the downregulation of FGFR1 by cortisol-treated ACM, which can bind to various gene promoters, reduced its capacity to bind to NLGN1 promoters, despite the presence of an FGFR1 binding to the NRXN3 promoter. Neuroligin is a synaptogenic transmembrane protein that recruits glutamate/GABA receptors for postsynaptic potentiation and consists of four subtypes; neuroligin 1 is usually localized in excitatory synapses whereas neuroligin 2/4 are at inhibitory synapses, and neuroligin 3 exists in both types of synapse. Specifically, neuroligin 1 prefers AMPAR to NMDAR and scaffolds GluR1/2 into PSD95, subsequently forming an excitatory synapse [47]. Altogether, the present study supports the suggestion that the inhibitory effect of cortisol-treated ACM on neuroligin 1 expression selectively inhibits glutamatergic synaptogenesis by reducing the recruitment of GluR1/2 into the synapse but neuroligin 2 expression is not involved in inhibiting glutamatergic synaptogenesis. However, many open questions about the particular effect of prenatal stress on neuroligin 1 remain. During early life, FGF2 and INFS contribute critically to neurogenesis, since many other mechanisms such as GR-dependent signaling are activated differently at this stage. In contrast, prolonged severe stress in adult rodents reduces LTP through the genomic pathway, whereas acute stress increases synaptic transmission via nongenomic pathways, both of which we found to be insignificant [48]. These effects were due to the differential expression and distribution of GR in the neonatal brain, because our results showed that knockdown of GR did not alter neuroligin 1 expression in differentiating neurons. Moreover, we could not observe significant changes to 
neuroligin 1 expression in fully differentiated neurons and adult mouse brain exposed to cortisol-treated ACM and corticosterone, respectively. These findings are supported by several reports comparing the effects of stress, depending on age. Chronic restraint stress in adulthood reduced neuroligin 2 but not neuroligin 1 expression in the hippocampus [49]. Another report also demonstrated that upregulation of neuroligin 1 did not induce synapse formation in adult-born dentate granule cells of the hippocampus [50]. The fact that neuroligin 1 is the most abundant isotype in the hippocampus also allows developing neurons from NSCs of the SGZ to rely heavily on it [51]. Collectively, these results support a unique mechanism susceptible to prenatal stress exists; in this present study, astrocytic FGF2-neuronal INFS axis that regulates neuroligin 1. Our in vivo data demonstrated that FGF2 restoration recovers neuroligin 1dependent glutamatergic synaptogenesis, inhibiting the progression of spatial memory loss, anxiety, and depressive mood. However, this approach neglects the potential or a systemic effect of FGF2 injection, which does not occur by overexpression of FGF2 in hippocampal astrocytes using AAV vectors; nevertheless, demonstrating the unique effects of prenatal stress on neuroligin 1 during early life can compensate for these limitations. In conclusion, to the best of our knowledge, our study is the first to identify neuroligin 1 as a potential target protein for treating prenatal glucocorticoid-induced neurogenesis defects and memory/mood disorders.

\section{Abbreviations}

ACM: astrocytic conditioned media; ALDH1L1: aldehyde dehydrogenase 1 family member L1; AMPA: aamino-3-hydroxy-5-methyl-4-isoxazole propionic acid; AMPAR: AMPA receptor; ChIP: chromatin immunoprecipitation; DCX: doublecortin ; E: embryonic day; FGF2: fibroblast growth factor 2; FGFR1: fibroblast growth factor receptor 1; GR: glucocorticoid receptor; GluR: ionotropic glutamate receptor; HPA: hypothalamus-pituitary-adrenal; INFS: integrative nuclear FGFR1 signaling; iPSC: induced pluripotent stem cell; LTP: long term potentiation; NGS: normal goat serum; NMDA: N-methyl-D-aspartate; NMDAR: NMDA receptor; NPC: neuroprogenitor cell; NSC: neural stem cell; NT: nontargetting; P: postnatal day; RNAPol: RNA polymerase; RTK: receptor tyrosine kinase; S.E.M.: standard error of mean; SGZ: subgranular zone; siRNA: small interfering RNA; SRRF: super-resolution radial fluctuations

\section{Declarations}

\section{Funding}

This research was supported by National R\&D Program through the National Research Foundation of Korea (NRF) funded by the Ministry of Science, ICT \& Future Planning (NRF-2020R1A2B5B02002442) and the Ministry of Education (2021R1I1A1A01047974).

\section{Competing interests}

No potential conflict of interest was reported by the all authors.

\section{Author contributions}


G.E.C. contributed to design of the work, performance of experiments, interpretation of data, and writing the paper.

C.W.C., M.R.P., J.H.Y., and Y.H.J. contributed to performance of experiments.

H.J.L. contributed to design of the work and interpretation of data.

H.J.H. contributed to design of the work, interpretation of data, and writing the paper.

\section{Data availability}

The authors declare that all the data supporting the findings of this study are available within this article, its supplementary information files, or are available from the corresponding author, who has all relevant data, upon reasonable request.

\section{Ethics approval}

The performance of animal study was designed in compliance with the ARRIVE guidelines. For animal study, all the experimental procedures were approved by the Institutional Animal Care and Use Committee of Seoul National University (SNU-190523-1-1, SNU-190917-6-1, SNU-200221-6-1).

\section{Consent to participate}

Not applicable.

\section{Consent to publish}

Not applicable.

\section{References}

1. Jeanneteau, F., et al., Bridging the gap between brain-derived neurotrophic factor and glucocorticoid effects on brain networks. Neuroendocrinology, 2019. 109(3): p. 277-284.

2. Anacker, C., et al., Role for the kinase SGK1 in stress, depression, and glucocorticoid effects on hippocampal neurogenesis. Proceedings of the National Academy of Sciences, 2013. 110(21): p. 87088713.

3. Brunson, K.L., et al., Long-term, progressive hippocampal cell loss and dysfunction induced by early-life administration of corticotropin-releasing hormone reproduce the effects of early-life stress. Proceedings of the National Academy of Sciences, 2001. 98(15): p. 8856-8861.

4. Provençal, N., et al., Glucocorticoid exposure during hippocampal neurogenesis primes future stress response by inducing changes in DNA methylation. Proceedings of the National Academy of Sciences, 2020. 117(38): p. 23280-23285. 
5. Chen, C.-C., et al., Selective activation of parvalbumin interneurons prevents stress-induced synapse loss and perceptual defects. Molecular psychiatry, 2018. 23(7): p. 1614-1625.

6. Noorlander, C.W., et al., Antenatal glucocorticoid treatment affects hippocampal development in mice. PloS one, 2014. 9(1): p. e85671.

7. Koutmani, Y. and K.P. Karalis, Neural stem cells respond to stress hormones: distinguishing beneficial from detrimental stress. Frontiers in physiology, 2015. 6: p. 77.

8. Egeland, M., P.A. Zunszain, and C.M. Pariante, Molecular mechanisms in the regulation of adult neurogenesis during stress. Nature reviews neuroscience, 2015. 16(4): p. 189-200.

9. Yuen, E.Y., et al., Repeated stress causes cognitive impairment by suppressing glutamate receptor expression and function in prefrontal cortex. Neuron, 2012. 73(5): p. 962-977.

10. Zhang, T.-Y., et al., Environmental enrichment increases transcriptional and epigenetic differentiation between mouse dorsal and ventral dentate gyrus. Nature communications, 2018. 9(1): p. 1-11.

11. Snyder, J.S., et al., Adult hippocampal neurogenesis buffers stress responses and depressive behaviour. Nature, 2011. 476(7361): p. 458-461.

12. Crossin, K.L., et al., Glucocorticoid receptor pathways are involved in the inhibition of astrocyte proliferation. Proceedings of the National Academy of Sciences, 1997. 94(6): p. 2687-2692.

13. Kirby, E.D., et al., Acute stress enhances adult rat hippocampal neurogenesis and activation of newborn neurons via secreted astrocytic FGF2. Elife, 2013. 2: p. e00362.

14. Turner, C.A., et al., Fibroblast growth factor-2 (FGF2) augmentation early in life alters hippocampal development and rescues the anxiety phenotype in vulnerable animals. Proceedings of the National Academy of Sciences, 2011. 108(19): p. 8021-8025.

15. Qian, X., et al., FGF2 concentration regulates the generation of neurons and glia from multipotent cortical stem cells. Neuron, 1997. 18(1): p. 81-93.

16. Teissier, A., et al., Early-life stress impairs postnatal oligodendrogenesis and adult emotional behaviour through activity-dependent mechanisms. Molecular psychiatry, 2020. 25(6): p. 1159-1174.

17. Kaech, S. and G. Banker, Culturing hippocampal neurons. Nature Protocols, 2006. 1(5): p. 2406-2415.

18. Wittenmayer, N., et al., Postsynaptic Neuroligin1 regulates presynaptic maturation. Proceedings of the National Academy of Sciences, 2009. 106(32): p. 13564-13569.

19. Choi, G.E., et al., BNIP3L/NIX-mediated mitophagy protects against glucocorticoid-induced synapse defects. Nature communications, 2021. 12(1): p. 1-18. 
20. Woodbury, M.E. and T. Ikezu, Fibroblast growth factor-2 signaling in neurogenesis and neurodegeneration. Journal of neuroimmune pharmacology, 2014. 9(2): p. 92-101.

21. Kiyota, T., et al., FGF2 gene transfer restores hippocampal functions in mouse models of Alzheimer's disease and has therapeutic implications for neurocognitive disorders. Proceedings of the National Academy of Sciences, 2011. 108(49): p. 1339-1348.

22. MacDonald, A.J., et al., Astrocytes in neuroendocrine systems: An overview. Journal of neuroendocrinology, 2019. 31(5): p. e12726.

23. Tyzack, G.E., et al., A neuroprotective astrocyte state is induced by neuronal signal EphB1 but fails in ALS models. Nature communications, 2017. 8(1): p. 1-17.

24. Clarke, L.E., et al., Normal aging induces A1-like astrocyte reactivity. Proceedings of the National Academy of Sciences, 2018. 115(8): p. 1896-1905.

25. Liddelow, S.A., et al., Neurotoxic reactive astrocytes are induced by activated microglia. Nature, 2017. 541(7638): p. 481-487.

26. Pearson-Leary, J., D.M. Osborne, and E.C. McNay, Role of glia in stress-induced enhancement and impairment of memory. Frontiers in integrative neuroscience, 2016. 9: p. 63.

27. Regnell, C.E., et al., Hippocampal adult neurogenesis is maintained by Neil3-dependent repair of oxidative DNA lesions in neural progenitor cells. Cell reports, 2012. 2(3): p. 503-510.

28. Sandi, C., Glucocorticoids act on glutamatergic pathways to affect memory processes. Trends in neurosciences, 2011. 34(4): p. 165-176.

29. Dunham-Ems, S.M., et al., Fibroblast growth factor receptor-1 (FGFR1) nuclear dynamics reveal a novel mechanism in transcription control. Molecular biology of the cell, 2009. 20(9): p. 2401-2412.

30. Terranova, C., et al., Global developmental gene programing involves a nuclear form of fibroblast growth factor receptor-1 (FGFR1). PloS one, 2015. 10(4): p. e0123380.

31. Fang, X., et al., Control of CREB-binding protein signaling by nuclear fibroblast growth factor receptor1: a novel mechanism of gene regulation. Journal of Biological Chemistry, 2005. 280(31): p. 2845128462.

32. Suri, D., et al., Early stress evokes age-dependent biphasic changes in hippocampal neurogenesis, BDNF expression, and cognition. Biological psychiatry, 2013. 73(7): p. 658-666.

33. Murthy, S. and E. Gould, How early life adversity influences defensive circuitry. Trends in neurosciences, 2020. 43(4): p. 200-212. 
34. Zhao, Y., et al., A mouse model of depression induced by repeated corticosterone injections. European journal of pharmacology, 2008. 581(1-2): p. 113-120.

35. Bakker, J.M., F. Van Bel, and C.J. Heijnen, Neonatal glucocorticoids and the developing brain: shortterm treatment with life-long consequences? Trends in neurosciences, 2001. 24(11): p. 649-653.

36. Vicini, S., The role of GABA and glutamate on adult neurogenesis. The Journal of physiology, 2008. 586: p. 3737.

37. Tyzio, R., et al., The establishment of GABAergic and glutamatergic synapses on CA1 pyramidal neurons is sequential and correlates with the development of the apical dendrite. Journal of neuroscience, 1999. 19(23): p. 10372-10382.

38. Raballo, R., et al., Basic fibroblast growth factor (Fgf2) is necessary for cell proliferation and neurogenesis in the developing cerebral cortex. Journal of neuroscience, 2000. 20(13): p. 5012-5023.

39. Knafo, S., et al., Facilitation of AMPA receptor synaptic delivery as a molecular mechanism for cognitive enhancement. PloS biology, 2012. 10(2): p. e1001262.

40. Huang, J.-Y., M.L. Miskus, and H.-C. Lu, FGF-FGFR mediates the activity-dependent dendritogenesis of layer IV neurons during barrel formation. Journal of neuroscience, 2017. 37(50): p. 12094-12105.

41. Park, M., AMPA receptor trafficking for postsynaptic potentiation. Frontiers in cellular neuroscience, 2018. 12: p. 361.

42. Gunn, B. and T. Baram, Stress and seizures: space, time and hippocampal circuits. Trends in neurosciences, 2017. 40(11): p. 667-679.

43. Iwata, T. and R.F. Hevner, Fibroblast growth factor signaling in development of the cerebral cortex. Development, growth \& differentiation, 2009. 51(3): p. 299-323.

44. Noda, M., et al., FGF-2 released from degenerating neurons exerts microglial-induced neuroprotection via FGFR3-ERK signaling pathway. Journal of neuroinflammation, 2014. 11(1): p. 1-11.

45. Saffell, J.L., et al., Expression of a dominant negative FGF receptor inhibits axonal growth and FGF receptor phosphorylation stimulated by CAMs. Neuron, 1997. 18(2): p. 231-242.

46. Chioni, A.-M. and R. Grose, FGFR1 cleavage and nuclear translocation regulates breast cancer cell behavior. Journal of cell biology, 2012. 197(6): p. 801-817.

47. Letellier, M., et al., A unique intracellular tyrosine in neuroligin-1 regulates AMPA receptor recruitment during synapse differentiation and potentiation. Nature communications, 2018. 9(1): p. 1-17.

48. Krugers, H.J., C.C. Hoogenraad, and L. Groc, Stress hormones and AMPA receptor trafficking in synaptic plasticity and memory. Nature reviews neuroscience, 2010. 11(10): p. 675. 
49. Van Der Kooij, M.A., et al., Impaired hippocampal neuroligin-2 function by chronic stress or synthetic peptide treatment is linked to social deficits and increased aggression. Neuropsychopharmacology, 2014. 39(5): p. 1148-1158.

50. Schnell, E., et al., Neuroligin-1 overexpression in newborn granule cells in vivo. PloS biology, 2012. 10: p. e48045.

51. Cheng, W., F. Han, and Y. Shi, Neonatal isolation modulates glucocorticoid-receptor function and synaptic plasticity of hippocampal and amygdala neurons in a rat model of single prolonged stress. Journal of affective disorders, 2019. 246: p. 682-694.

\section{Figures}

\section{Figure 1}

Prenatal stress triggers anxiety/depression-like behavior and spatial memory dysfunction by suppressing neurogenesis in mice. $(\mathbf{a}-\mathbf{f})$ Four weeks after exposure to maternal vehicle or corticosterone $(10 \mathrm{mg} / \mathrm{kg})$ at E14, mice underwent behavior tests, dexamethasone suppression test, and were sacrificed for IHC at P23. (a) Open field test was performed to assess anxiety-like behavior for $10 \mathrm{~min}$. Relative time at the periphery or center and total distance explored were determined. $n=6$. (b) Mice were presented to the forced swim test for $6 \mathrm{~min}$. Immobile and mobile activities were determined during the last $4 \mathrm{~min}$. $n=5$. (c) The mice were subjected to Y-maze test to evaluate spatial memory function. $n=6$. (d) Before the dexamethasone suppression test, the blood was collected. Blood was extracted again $1 \mathrm{~h}$ after dexamethasone $(50 \mu \mathrm{l} / \mathrm{kg})$ was injected. Plasma corticosterone was determined by ELISA. $n=5$. \#\# indicates $p<0.01$ versus plasma corticosterone of dexamethasone-injected control mice. (e-f) Slide samples of both dorsal and ventral hippocampus for $\mathrm{IHC}$ were collected and the quantification analysis was performed in subgranular zone (SGZ). (e) The hippocampus was immunostained with BrdU (green), c-Fos (red), and DAPI (blue). Scale bars, $100 \mu \mathrm{m}$ (magnification, $\times 200) . n=5$. (f) The hippocampus was immunostained with NeuN (green), DCX (red), and DAPI (blue). Scale bars, $100 \mu \mathrm{m}$ (magnification, $\times 200$ ). $n=5$. All immunofluorescence images are representative. $n=5$ from each animal with two technical replicates in results of IHC. Quantitative data are presented as a mean \pm S.E.M. The representative images were acquired by SRRF imaging system. * ** indicates $p<0.05$ and $p<0.01$ versus control, respectively.

\section{Figure 2}

Glucocorticoid impairs neurogenesis through activating A1-like astrocytes. (a-b) After exposed to prenatal vehicle or corticosterone (10 mg/kg) at E14, the hippocampus from mice at P1 was collected. (a) The 
cells dissociated from the hippocampus were immunostained with BrdU (green) and c-Fos (red). The ratio of c-Fos positive cells to BrdU positive cells was analyzed by flowcytometer. $n=5$. (b) The cells dissociated from the hippocampus were immunostained with DCX (green) and NeuN (red). The ratio of DCX positive cells to NeuN positive cells was analyzed by flowcytometer. $n=5$. (c-d) Human NSCs were incubated with astrocytic differentiation media with cortisol $(1 \mu \mathrm{M})$ for 5 days. (c) The percentages of apoptotic cells (Annexin V positive cells) were analyzed by Annexin V/PI analysis, measured by flowcytometer. $n=5$. (d) The differentiated cells were immunostained with GFAP (green) and PI (red). The ratio of GFAP positive cells to PI positive cells was analyzed by flowcytometer. $n=5$. (ef) Human NSCs were incubated with astrocytic differentiation media for 5 days and then cortisol $(1 \mu \mathrm{M})$ was treated for $48 \mathrm{~h}$. (e) Astrocytes were immunostained with GFAP (green) and DAPI (blue). Astrocytic surface area was analyzed by Image J. Scale bars, $20 \mu \mathrm{m}$ (magnification, $\times 1,000$ ). $n=5$. (f) The expressions of GFAP, ALDH1L1, STAT3, C3, and $\beta$-actin were detected by western blot. $n=5$. (g-h) Human NSCs were incubated with astrocytic differentiation media for 5 days and cortisol $(1 \mu \mathrm{M})$ was treated for $48 \mathrm{~h}$. Then, the ACM were collected and administered to the human NSCs incubated with neuronal differentiation media for 5 days. After 5 days of treatment, the neurons were immunostained as appropriate. (g) The cells were immunostained with BrdU (green) and c-Fos (red). The ratio of c-Fos positive cells to BrdU positive cells was analyzed by flowcytometer. $n=5$. (h) The cells were immunostained with DCX (green) and NeuN (red). The ratio of DCX positive cells to NeuN positive cells was analyzed by flowcytometer. $n=5$. All immunofluorescence images are representative. $n=5$ from independent experiments with two technical replicates each. Quantitative data are presented as a mean \pm S.E.M. The representative images were acquired by SRRF imaging system. ${ }^{*}{ }^{* *}$ indicates $p<0.05$ and $p<0.01$ versus control, respectively.

\section{Figure 3}

Glucocorticoid-induced change in astrocyte homeostasis inhibits glutamatergic synaptogenesis. $(a-b, d-g)$ Five days after astrocytic differentiation of human NSCs, cortisol $(1 \mu \mathrm{M})$ was treated for $48 \mathrm{~h}$ and ACM were collected. Human NSCs were cultured in neuronal differentiation media for 5 days and ACM were treated for $48 \mathrm{~h}$, as appropriate. (a) Conditioned neurons were stained with FM4-64 dye and stimulated with high $\mathrm{K}^{+}$buffer for destaining. Time-lapse imaging was performed over $90 \mathrm{sec}$ at $1 \mathrm{sec}$ intervals with an Eclipse Ts2 ${ }^{\mathrm{TM}}$ fluorescence microscopy. $n=5$. (b) (Upper images) The visualization of vGLUT1 (green), PSD95 (red), and DAPI (blue) was done in conditioned neurons. (Lower images) The detection of vGAT (green), PSD95 (red) and DAPI (blue) was performed in conditioned neurons. Scale bars, $20 \mu \mathrm{m}$ (magnification, $\times 1,000) . n=5$. (c) After exposed to prenatal vehicle or corticosterone $(10 \mathrm{mg} / \mathrm{kg})$ at E14, the hippocampus from mice at P1 was collected and underwent fraction to gain synaptosome. The expressions of GluR1/2, NR1, NR2B, a-tubulin, and PSD95 were detected by western blot. The a-tubulin and PSD95 were used as a loading control for cytosol and synaptosome, respectively. $n=5$. (d) (Left) PSD 95 (green), GluR1/2 (red), and DAPI (blue) were visualized through immunostaining. (Right) PSD95 (green), NR1 or NR2B (red), and DAPI (blue) were visualized through immunostaining. Scale bars, $20 \mu \mathrm{m}$ 
(magnification, $\times 1,000) . n=5$. (e) The expressions of GluR1/2, a-tubulin, and synaptophysin in cytosol, synaptosome, and total lysates were detected by western blot. The a-tubulin and synaptophysin were used as a loading control for cytosol and synaptosome, respectively. $n=5$. (f) Before measuring kinetics of FM4-64 dye, AMPA $(100 \mu \mathrm{M})$ and NMDA $(100 \mu \mathrm{M})$ were pretreated as appropriate. Then, neurons were stimulated with high $\mathrm{K}^{+}$buffer for destaining. Time-lapse imaging was performed over $90 \mathrm{sec}$ at $1 \mathrm{sec}$ intervals with an Eclipse Ts2 ${ }^{\mathrm{TM}}$ fluorescence microscopy. $n=5$. (g) Conditioned neurons were immunostained with MAP2 (green) and DAPI (blue). The Sholl analysis was performed for analyzing neurite outgrowth. Scale bars, $50 \mu \mathrm{m}$ (magnification, $\times 400$ ). $n=5$. All immunofluorescence images are representative. $n=5$ from independent experiments with two technical replicates each. Quantitative data are presented as a mean \pm S.E.M. The representative images were acquired by SRRF imaging system. * ** indicates $p<0.05$ and $p<0.01$ versus control, respectively.

\section{Figure 4}

Glucocorticoid suppresses by reducing astrocytic HMW FGF2 and its downstream INFS. (a) After exposed to prenatal vehicle or corticosterone $(10 \mathrm{mg} / \mathrm{kg})$ at E14, the hippocampus from mice at P1 was collected. RNA sequencing was performed using RNA extracted from the hippocampal tissue. Scatter plot represents the log-fold changes between the mice exposed to prenatal vehicle (control) and the mice exposed to prenatal corticosterone. $n=3$. (b-c) Five days after astrocytic differentiation of human NSCs, cortisol $(1 \mu \mathrm{M})$ was treated for $48 \mathrm{~h}$. The media were then collected. (b) The growth factor antibody array was performed using the extracted media. $n=5$. (c) Human FGF2 levels were measured using ELISA. $n=5$. $(\mathrm{d}-\mathrm{g})$ Five days after astrocytic differentiation of human NSCs, cortisol $(1 \mu \mathrm{M})$ was treated for $48 \mathrm{~h}$ and ACM were collected. (d) The neurons differentiated from human NSCs (DIV 7) were treated with ACM and FGF2 (2 ng/ml) for 5 days as appropriate. Then, the cells were immunostained with DCX (green) and NeuN (red). The ratio of DCX positive cells to NeuN positive cells was analyzed by flowcytometer. $n=5$. (eg) The neurons (DIV 7) were treated with ACM and FGF2 for $24 \mathrm{~h}$ as appropriate. (e) Protein levels of FGFR1 in subcellular fraction samples were detected by western blotting. The $\beta$-actin was used as a loading control for cytosol. The lamin $\mathrm{A} / \mathrm{C}$ and pancadherin were used as a loading control for nucleus and membrane, respectively. $n=5$. (f) The cells were immunostained with FGF2 (green), FGFR1 (red), and DAPI (blue). Pearson's correlation coefficient between FGF2 and FGFR1 in nuclear region was quantified. Scale bars, $20 \mu \mathrm{m}$ (magnification, $\times 1,000$ ). $n=5$. (g) Subcellular fraction was done and FGFR1 was coimmunoprecipiated with FGF2 in nuclear parts. HMW FGF2 levels in immunoprecipitated samples were quantified. $n=5$. All immunofluorescence images are representative. $n=5$ from independent experiments with two technical replicates each. Quantitative data are presented as a mean \pm S.E.M. The representative images were acquired by SRRF imaging system. ${ }^{* *}$ indicates $p<0.01$ versus control and ${ }^{\# \#}$ indicates $p<0.01$ versus cortisol, respectively. 


\section{Figure 5}

Reduction in INFS by cortisol inhibits AMPA-mediated glutamatergic synaptogenesis. (a-d) Five days after astrocytic differentiation of human NSCs, cortisol $(1 \mu \mathrm{M})$ was treated for $48 \mathrm{~h}$ and ACM were collected. The neurons differentiated from human NSCs (DIV 6) were transfected with pcDNA3.1/c-eGFP or pcDNA3.1/FGFR1-C-eGFP for $24 \mathrm{~h}$ prior to ACM treatment for $48 \mathrm{~h}$. Then, FGFR1 antibody $(100 \mu \mathrm{g} / \mathrm{ml})$ was pretreated for $1 \mathrm{~h}$ prior to ACM treatment for $48 \mathrm{~h}$. (a-b) The cells were immunostained with GluR1/2 (blue) and PSD 95 (red). Pearson's correlation coefficient between GluR1/2 and PSD95 was quantified. Scale bars, $20 \mu \mathrm{m}$ (magnification, $\times 1,000$ ). $n=5$. (c) The neurons were stimulated with high $\mathrm{K}^{+}$buffer for destaining. Time-lapse imaging was performed over $90 \mathrm{sec}$ at $1 \mathrm{sec}$ intervals with an Eclipse Ts2 ${ }^{\mathrm{TM}}$ fluorescence microscopy. $n=5$. (d) Conditioned neurons were immunostained with MAP2 (red) and DAPI (blue). The Sholl analysis was performed for analyzing neurite outgrowth. Scale bars, $50 \mu \mathrm{m}$ (magnification, $\times 400$ ). $n=5$. All immunofluorescence images are representative. $n=5$ from independent experiments with two technical replicates each. Quantitative data are presented as a mean \pm S.E.M. The representative images were acquired by SRRF imaging system. ${ }^{*}{ }^{* *}$ indicates $p<0.05, p<0.01$ versus

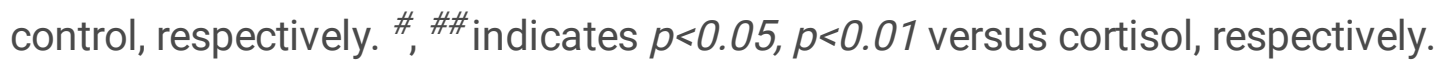

\section{Figure 6}

Suppressed INFS by cortisol downregulates neuroligin 1 and subsequent neurogenesis. (a-h) Five days after astrocytic differentiation of human NSCs, cortisol $(1 \mu \mathrm{M})$ was treated for $48 \mathrm{~h}$ and ACM were collected. (a-b) The neurons differentiated from human NSCs (DIV 7) were incubated with ACM as appropriate for $24 \mathrm{~h}$ (a) The mRNA expressions were analyzed by real-time PCR. $n=5$. (b) DNA was immunoprecipitated with anti-IgG, RNA polymerase, and FGFR1 antibody. The samples were amplified with primers of GAPDH, NLGN1, NLGN3, NRXN2, and NRXN3 gene. $n=5$. (c) The neurons differentiated from human NSCs (DIV 6) were transfected with pcDNA3.1/c-eGFP or pcDNA3.1/FGFR1-c-eGFP for $24 \mathrm{~h}$ prior to ACM treatment for $48 \mathrm{~h}$. Actinomycin $\mathrm{D}(500 \mathrm{ng} / \mathrm{ml})$ was pretreated for 30 min before ACM treatment as appropriate. Expression levels of neuroligin 1 was detected by western blotting. $\$ \$$ indicates, $p<0.01$ versus cortisol with pcDNA3.1/FGFR1-c-eGFP transfection. $n=5$. (d-g) The neurons differentiated from human NSCs (DIV 6) were transfected with pcDNA3.1/c-eGFP or pcDNA3.1/NLGN1-c-eGFP for $24 \mathrm{~h}$ prior to ACM treatment. (d-f) The ACM were administered to conditioned neurons for $48 \mathrm{~h}$. (d) The neurons were immunostained with GluR1/2 (blue) and PSD95 (red). Pearson's correlation coefficient between GluR1/2 and PSD 95 was quantified. Scale bars, $20 \mu \mathrm{m}$ (magnification, $\times 1,000$ ). $n=5$. (e) The neurons were stimulated with high $\mathrm{K}^{+}$buffer for destaining. Time-lapse imaging was performed over 90 sec at $1 \mathrm{sec}$ intervals with an Eclipse Ts2 ${ }^{\mathrm{TM}}$ fluorescence microscopy. $n=5$. (f) The neurons were immunostained with MAP2 (red) and DAPI (blue). The Sholl analysis was performed for analyzing neurite outgrowth. Scale bars, $50 \mu \mathrm{m}$ (magnification, 400). $n=5$. (g) ACM were administered to the conditioned neurons for 5 days. The neurons were then immunostained with DCX (green) and NeuN (red). The ratio of 
DCX positive cells to NeuN positive cells was analyzed by flowcytometer. $n=5$. (h) The differentiated neurons (DIV 7 or 21) were treated with ACM as appropriate. Neuroligin 1 expressions were detected by western blotting. $n=5$. All immunofluorescence images are representative. $n=5$ from independent experiments with two technical replicates each. Quantitative data are presented as a mean \pm S.E.M. The representative images were acquired by SRRF imaging system. ${ }^{*}{ }^{* *}$ indicates $p<0.05, p<0.01$ versus control, respectively. \#, \#\# indicates $p<0.05, p<0.01$ versus cortisol, respectively.

\section{Figure 7}

\section{Restoration of neuroligin 1 by FGF2 pretreatment reverses the deleterious effects of corticosterone on glutamatergic synapse formation and hippocampal-related behaviors. (a) The E14 or adult mice (8-week-} old) were exposed to corticosterone (10 mg/kg). Five days after treatment, hippocampus was collected and neuroligin 1 was detected by western blotting of its lysates. $n=5$. (b-g) After exposure to maternal vehicle or corticosterone (10 mg/kg) at E14 and pretreatment of FGF2 starting at P1, P23 mice underwent behavior tests and were sacrificed (b) Slide samples of both dorsal and ventral hippocampus for IHC were collected and hippocampal tissue was immunostained with GluR1/2 (green), PSD95 (red), and DAPI (blue). Pearson's correlation coefficient between GluR1/2 and PSD95 was quantified. Scale bars, $100 \mu \mathrm{m}$ (magnification, $\times 200$ ). $n=5$. (c) The hippocampal tissue underwent subcellular fraction to gain synaptosome. The expressions of GluR1/2, PSD95, and a-tubulin were detected by western blot. PSD95 and a-tubulin were used as a loading control for synaptosome and cytosol, respectively. $n=5$. (d) PSD95 was co-immunoprecipitated with GluR1/2 and neuroligin 1 in synaptosome. $n=5$. (e) Open field test was performed. Relative time at the periphery or center and total distance explored were determined. $n=5$. ( $f$ ) Mice were presented to the forced swim test for $6 \mathrm{~min}$. Immobile and mobile activities were determined during the last 4 min. $n=5$. (g) The mice were subjected to Y-maze test to evaluate spatial memory function. $n=6$. All immunofluorescence images are representative. $n=5$ or 6 from each animal with two technical replicates in results of $\mathrm{IHC}$ or western blotting. Quantitative data are presented as a mean \pm S.E.M. The representative images were acquired by SRRF imaging system. ${ }^{* \star}$ indicates $p<0.01$ versus

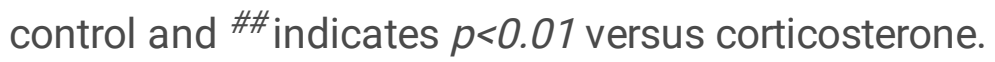

\section{Figure 8}

\section{The schematic model for mechanisms of inhibition in neuroligin 1-dependent neurogenesis by prenatal glucocorticoid was shown.}

Astrocytic FGF2 and downstream nuclear neuronal FGFR1 trigger neuroligin 1 expression, which mainly causes glutamatergic synaptogenesis. However, prenatal glucocorticoid transforms astrocytes into A1like reactive astrocytes and reduces FGF2 release, resulting in impaired synaptogenesis and subsequent neurogenesis. 


\section{Supplementary Files}

This is a list of supplementary files associated with this preprint. Click to download.

- SupplementaryFigure4th.pdf 